\section{Tampon-induced toxic shock syndrome}

Sir,

Since the definition of the toxic shock syndrome (TSS) in 1980 , menstruation has been associated with the condition in about $50 \%$ of cases. ' The number of reported cases has declined in recent years but we describe a recent fatal case at our hospital that serves to remind that tamponrelated TSS has yet to be completely combated.

A 29 year old woman with a known history of schizophrenia and intravenous drug abuse presented in an acute confusional state. She had fallen 3 days earlier after taking an overdose of haloperidol and benzodiazepines and laid on the floor during that period. Vigilant neighbours were alerted and brought her into hospital.

Clinically she was confused, pyrexial and dehydrated. Her skin had a mottled, dusky appearance. Cardiovascular examination revealed a regular tachycardia of $140 /$ minute and a blood pressure of $130 / 90 \mathrm{mmHg}$. Respiratory examination revealed right basal crepitations. Her left leg was swollen and erythematous with some superficial bruising compatible with her recent fall.

Initial blood tests showed a haemoglobin of $18.8 \mathrm{~g} / \mathrm{dl}$, white cells $30.2 \times 10 / 1$ (neutrophilia), platelets $361 \times 10 /$ 1 , sodium $131 \mathrm{mmol} / 1$, potassium $5.1 \mathrm{mmol} / \mathrm{l}$, urea $15.8 \mathrm{mmol} / \mathrm{l}$, glucose $9.2 \mathrm{mmol} / \mathrm{l}$ and creatinine $313 \mathrm{mmol} / \mathrm{l}$. Electrocardiograph revealed sinus tachycardia of 140/minute and chest X-ray was unremarkable.

Nurses discovered the presence of a retained vaginal tampon that was soaked in pus and very offensive. The presumed diagnosis was septicaemia secondary to an infected tampon. She was started on intravenous cefotaxime and metronidazole (there was a strong suspicion of penicillin allergy) and given intravenous fluids.

The following morning she suddenly suffered an asystolic cardiorespiratory arrest, but with prolonged cardiac massage and repeated boluses of adrenaline and atropine a circulation was restored. Chest X-ray now revealed a mottled appearance compatible with the adult respiratory distress syndrome (ARDS). She required ventilatory and inotropic support. However, she became increasingly anuric, hypotensive and unresponsive. She died 24 hours later.

Subsequent investigations revealed a heavy growth of Staphylococcus aureus from the infected tampon and from multiple blood cultures. This isolate belonging to phage group III produced enterotoxins $A$ and $D$. At necropsy it was concluded that she died from sepsis related to the infected tampon.

The complicating factors of drug overdose, psychotic illness and recent fall no doubt contributed to the delay in medical care reaching our patient. The clinical scenario rapidly culminated in a cardio-respiratory arrest and then death despite antibiotics, intensive cardiovascular support and ventilation.

Although TSST-1 toxin produced by phage group I strains of $S$. aureus is classically associated with tamponinduced TSS, ${ }^{2}$ a number of other enterotoxins have been demonstrated and may be of greater virulence in contributing to a higher mortality in this condition. ${ }^{3,4}$

TSS should be a notifiable disease to ensure proper surveillance of the condition and our case comes at a time when there is a review by the Department of Health of notifiable diseases. There are about 20 cases of confirmed or probable TSS identified each year in the UK, not all of them associated with menstruation, and with 2-4 deaths. ${ }^{5}$ There were only five reported menstrual deaths in the years $1985-90 .^{6}$

TSS, with or without menstruation, needs early recognition and prompt aggressive therapy to avoid, be it very rare, a fatal outcome. Death from TSS associated with a Staphylococcus aureus producing enterotoxins $A$ and $D$ is very unusual.

Sandeep Gupta
Anju Sahdev
Sarah Forsythe
Paul Wright'
Richard Wray
Departments of Medicine and 'Microbiology,
Conquest Hospital,
The Ridge,
St Leonards on Sea,

East Sussex TN35 7RD, UK.

\section{References}

1. Davies, J.P., Chesney, P.J., Wand, P.J. \& LaVenture, M. Toxic shock syndrome, epidemiological features, recurrence risk factors and prevention. $N$ Engl J Med 1980, 303: 1429-1435.

2. Schlievert, P.M., Shands, K.N., Dan, B.B., Schmid, G.P. \& Nishimura, R.D. Identification and characterisation of an exotoxin from Staphylococcus aureus associated with toxicshock syndrome. J Infect Dis 1981, 143: 509-516.

3. Williams, G.R. The toxic shock syndrome. Br Med J 1990, 300: 960.

4. Bergdoll, M.S., Crass, B.A., Reiser, R.F., Robbins, R.N. \& Davis, J.P. A new staphylococcal enterotoxin, enterotoxin F, associated with toxic-shock syndrome. Staphylococcus aureus isolates. Lancet 1981, i: 1017-1021.

5. De Saxe, M.J., Hawtin, P. \& Wieneke, A.A. Toxic shock syndrome in Britain-epidemiology and microbiology. Postgrad Med J 1986, 61 (Suppl): 5-21.

6. Marples, R.R. \& Wieneke, A.A. Toxic Shock Syndrome in the UK: 1985-1990. Communicable Disease Report 1991, 1: R91-R92. 